European Federation of Societies for Ultrasound in Medicine and Biology

\title{
Learning by playing - using computer games to enhance ultrasound education
}

In ultrasound education, there is often a lack of hands-on experience due to the limited availability of experienced tutors, patients, volunteers, and ultrasound machines. Simulators can substitute training with real humans to some extent, but they are not widely used, mainly because of their costs. Playing a computer game might be a way to add to the training opportunities in ultrasound education. An example for such a game is the SonoGame that has been developed as part of a medical imaging research project with the help of computer science students from the Flensburg University of Applied Sciences in Germany. This simulation-based game requires only a standard computer and off-the-shelf gaming hardware, thus making it widely deployable.

The basic idea of the SonoGame is to provide the trainee with a step-by-step introduction to ultrasound in a playful way, first focusing on basic physics, spatial understanding and hand-eye coordination, and later addressing anatomical aspects. To achieve this, different mini games are pres- ented to the user. In each of these mini games, one first has to examine artificial volumes that contain simple geometric objects like spheres, tubes and cubes. An off-the-shelf $3 \mathrm{D}$ game controller serves as virtual ultrasound probe. As a more realistic and similarly inexpensive alternative, a $3 \mathrm{D}$ printed probe (or a case from an unused real ultrasound probe) can be equipped with a motion tracking device known from consumer virtual reality solutions.

By moving this virtual probe like a real ultrasound probe, the trainee generates slice images from the virtual volumes. With an additional $2 \mathrm{D}$ joystick, the volume can be rotated. In one of the mini games, one has to find out which of four given objects one is currently examining $\mathbf{F i g . 1 a ) . ~ I n ~ a n o t h e r ~}$ mini game, one needs to find out which of four given slice images belongs to the object one is currently examining ( $\nabla$ Fig. 1b). In a more advanced, quite challenging mini game, the user needs to recreate a given slice image within a certain tolerance ( $\triangleright$ Fig. 1c). To achieve different levels of difficulty within a mini game, the slice images can be displayed in different ways:

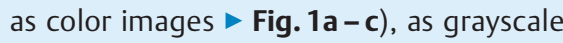
images ( $\triangleright$ Fig. $1 \mathbf{d}$ ) or more like ultrasound images, emphasizing object boundaries and including imaging artifacts like shadowing ( $\triangleright$ Fig. $\mathbf{1 e}-\mathbf{h}$ ).

By solving the gaming tasks, the trainee collects points and can try to beat the high score, sometimes while competing against time. With more points, more advanced levels are unlocked that contain increasingly complex volumes to be examined. In later levels, also anatomical objects are used, either in a schematic way ( $\triangleright$ Fig. 1i, illustrating a task focusing on arm nerves) or by including computed tomography data sets ( $\triangleright$ Fig. 1j, abdominal scan).

The SonoGame has been used at several workshops and hands-on exhibits, e.g. at the Ultraschall 2017 and 2018 meetings of the Austrian, German and Swiss Societies for Ultrasound in Medicine. Participants clearly appreciated the "fun factor" of this
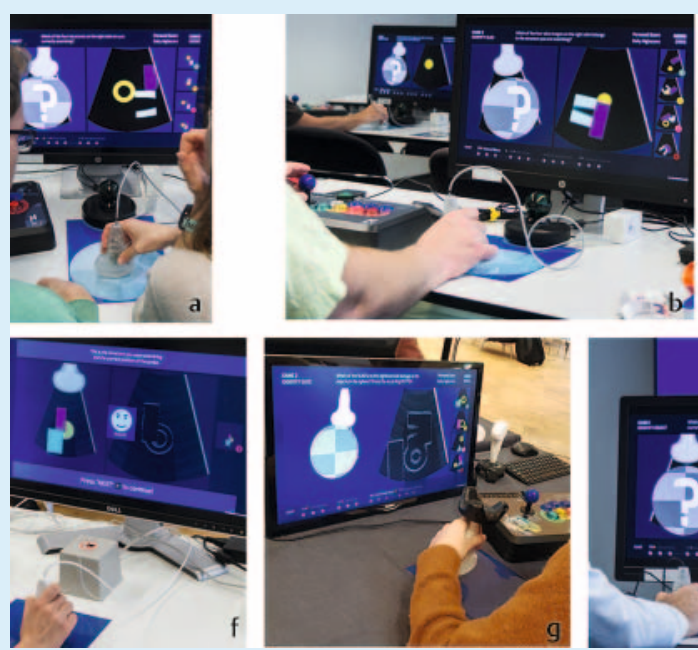
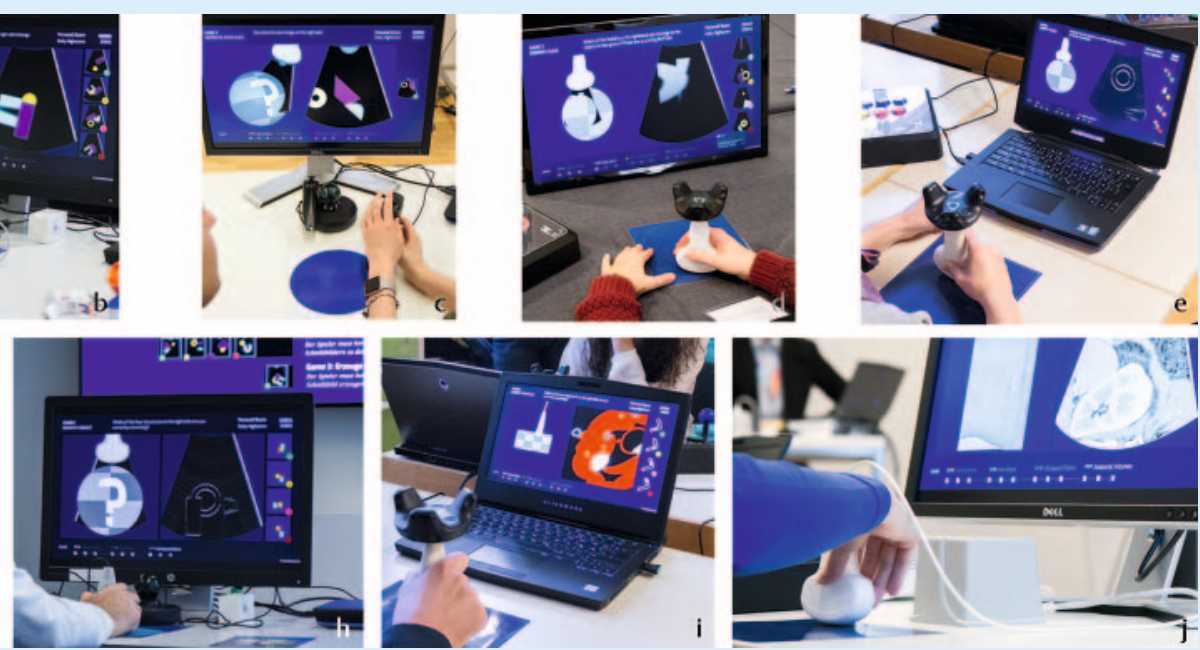

> Fig. 1 
approach, found it motivating and confirmed its usefulness as it addresses basic skills needed for ultrasound imaging, particularly spatial understanding and the handling of the probe. It was often emphasized that such a game could be an easily deployable and well-appreciated additional offer in ultrasound education for students and physicians in training. As the SonoGame represents an inexpensive approach, its use in skills labs with multiple, readily available workstations was envisioned. In such a lab, one could better prepare oneself for subsequent (limited) training with real patients or volunteers, thus making the rather rare hands-on experiences potentially more effi- cient and the overall training experience “more complete". Some would even like to use it at home.

In any case, users of the SonoGame regularly had a lot of fun. If there would be a "try not to laugh or smile" challenge for ultrasound education approaches (and not for YouTube videos, for a change), chances are the SonoGame would be in the finals.

In the meantime, at the Flensburg University of Applied Sciences, the students are eager to apply their acquired skills in programming, game design, computer graphics and virtual reality to take the SonoGame to the next level. More mini games, a mobile version for Android and iPhones and a virtual reality solution utilizing head-mounted displays are currently being developed.

Prof Dr. Michael Teistler

Professor of Computer Science at the Flensburg University of Applied Sciences

teistler@hs-flensburg.de

The development of the SonoGame has been supported by a research grant of the Marga und Walter Boll Stiftung (non-profit foundation) from Kerpen, Germany. 